\title{
Cinética de secagem em camada de espuma da polpa da manga cv. Haden
}

\author{
Edmilson Dantas da Silva Filho'*, Rossana Maria Feitosa de Figueirêdo \\ Alexandre José de Melo Queiroz², Mary Karlla Araújo Guimarães' \\ 'Instituto Federal de Educação, Ciência e Tecnologia da Paraíba, Campina Grande, PB, Brasil \\ ${ }^{2}$ Universidade Federal de Campina Grande, Campina Grande, PB, Brasil \\ *Autor correspondente, e-mail: edmsegundo@hotmail.com
}

\section{Resumo}

A secagem em camada de espuma é um processo econômico baseado na formação de uma espuma estável através do batimento da matéria-prima com promotores de espuma e que se obtém após a secagem um produto em pó. Com isso, o presente trabalho teve como objetivo estudar a cinética de secagem da polpa da manga $\mathrm{cv}$. Haden pelo método de secagem em camada de espuma nas temperaturas de 50,60 e $70^{\circ} \mathrm{C}$ e com três diferentes espessuras da camada $(0,5 ; 1,0 \mathrm{e}$ $1,5 \mathrm{~cm}$ ). Os modelos matemáticos de Henderson \& Pabis, Henderson e Logarítmico foram ajustados aos dados experimentais das secagens, adotando-se como critério de avaliação dos modelos o coeficiente de determinação $\left(R^{2}\right)$ e o desvio quadrático médio (DQM). Dos experimentos de secagens realizados foi observado que quanto menor a espessura da camada de espuma e maior a temperatura do ar de secagem menor é o tempo de secagem. Os menores tempos de secagem ocorreram na temperatura de $70^{\circ} \mathrm{C}$, com os tempos de 480,660 e 780 minutos, para as espessuras de 0,$5 ; 1,0$ e 1,5 cm, respectivamente. Os modelos de Henderson \& Pabis, Henderson e Logarítmico podem ser utilizados para representar o processo de secagem em razão de terem apresentado altos coeficientes de determinação $\left(R^{2}\right)$ e baixos desvios quadráticos médios (DQM).

Palavra chave: Mangifera indica L, polpa, secagem

\section{Drying kinetics in foam layer of mango flesh cv. Haden}

\begin{abstract}
Foam mat drying is an economical process based on the formation of a stable foam by beating the raw material with foam promoters, obtaining after drying a product in a powder form. Thus, the present study aimed to evaluate the drying kinetics of mango flesh cv. Haden with the foam mat drying method at drying temperatures of 50,60 and $70^{\circ} \mathrm{C}$, with three different thickness of the layer $(0.5,1.0$ and $1.5 \mathrm{~cm})$. The mathematical models of Henderson \& Pabis, Henderson and Logarithmic were fitted to experimental data and were used as criteria for evaluating the models the coefficient of determination $\left(R^{2}\right)$ and the root mean square deviation (DQM). It was observed that smaller thickness of the foam mat and higher air temperature decreased the drying time. Short drying time occurred at $70^{\circ} \mathrm{C}$, with 480,660 and 780 minutes for the thickness of $0.5 ; 1.0$ and $1.5 \mathrm{~cm}$, respectively. The Henderson \& Pabis, Henderson and Logarithmic models can be used to represent the drying process since high coefficients of determination $\left(R^{2}\right)$ and lower root mean square deviation (DQM) were observed.
\end{abstract}

Key words: Mangifera indica L, flesh, drying 


\section{Introdução}

Dentre as principais frutas produzidas no Brasil tem-se a manga, com uma produção em 2012 de 1.175.735 toneladas em uma área plantada de 73.690 ha, com rendimento médio de 16.038 quilos por hectares conforme dados do IBGE (2013).

Dentre os principais nutrientes encontrados em sua composição, tem-se: os carboidratos, as fibras, as vitaminas e os minerais (KUSKOSKI et al., 2006).

Os principais produtores de manga no país são os Estados do Nordeste, com cerca de 51.712 hectares de área colhida correspondendo a $67,7 \%$ da área total, sendo o Estado da Bahia o maior produtor desta região seguida de Pernambuco (IBGE, 2011 1). As perdas de frutas em países em desenvolvimento giram em torno de 10 a 40\% o que justifica a utilização de processos capazes de conservar tais produtos por um tempo maior (EL-AOUAR e MURR, 2003).

A vida útil pós-colheita da maioria dos frutos é muito curta, principalmente, quando colhidos maduros e armazenados em temperatura ambiente. Diante disso, um dos processos mais utilizados para aumentar a vida útil é a secagem. As vantagens de se utilizar o processo de secagem são várias, dentre as quais: a facilidade na conservação do produto: a estabilidade dos componentes aromáticos à temperatura ambiente por longos períodos de tempo; a proteção contra degradação enzimática e oxidativa; a redução da massa do produto; a economia de energia por não necessitar de refrigeração e a disponibilidade do produto durante qualquer época do ano (FEMENIA et al., 2009).

Atualmente, os produtos em pó, obtidos a partir de polpas de frutas, são cada vez mais utilizados pela indústria nacional de alimentos, uma vez que o processamento desses frutos reduz significativamente os custos com embalagens, transporte, armazenamento e conservação (COSTA et al., 2003).

Dentre as técnicas empregadas para a obtenção de produtos alimentícios em pó, a secagem em camada de espuma (foam mat drying), destaca-se por ser um método em que alimentos líquidos ou semilíquidos são transformados em espumas estáveis através de vigorosa agitação e incorporação de agentes espumantes para, posteriormente, serem desidratados (SILVA et al., 2008).

A secagem em camada de espuma é considerada um processo simples e de baixo custo que apresenta a particularidade de utilizar agentes que tem a função de facilitar e manter a estabilidade da espuma durante o processo. Tem como vantagens $\circ$ processamento em baixas temperaturas, menor tempo de desidratação devido à maior área de contato exposta ao ar, remoção mais rápida da água do produto e obtenção de um produto poroso facilmente reidratável.

Este trabalho teve como objetivo avaliar o estudo da cinética de secagem da polpa da manga cv. Haden, pelo método de secagem em camada de espuma em diferentes temperaturas e espessuras da camada de espuma.

\section{Material e Métodos}

As mangas utilizadas foram da variedade Haden, produzidas no Vale do São Francisco, no Perímetro Irrigado Senador Nilo Coelho, Petrolina, PE. Após a aquisição, as mangas foram transportadas em caixas de madeira adequadas até o local de processamento. Inicialmente, os frutos foram selecionados manualmente observando-se critérios de uniformidade do grau de maturação e integridade física, devendo apresentar-se em estádio de maturação ideal para consumo. No material selecionado foi feito uma pré-lavagem e lavagem em água clorada (40 ppm de cloro ativo/15min) posteriormente, foram enxaguados em água corrente a fim de se retirar o excesso de cloro, sendo realizada em água corrente da rede de abastecimento pública. Os frutos foram descascados manualmente com faca de aço inoxidável e em seguida ocorreram às etapas de despolpamento e refinamento. Após a etapa do refinamento, a polpa foi embalada em sacos de polietileno de baixa densidade com volume de 1 litro (dimensão $10 \times 25 \mathrm{~cm}$ ) e em seguida congelada por imersão em uma produtora de picolé a $-20^{\circ} \mathrm{C}$ e armazenada em freezer a $-18^{\circ} \mathrm{C}$ até o momento da realização dos experimentos.

A elaboração da espuma foi feita 
em batedeira doméstica pela mistura e homogeneização da polpa da manga Haden com adição de $1,5 \%$ de Super Liga Neutraß e $1,5 \%$ de Emustab ${ }^{\circledR}$ durante 30 minutos de batimento. Em seguida, a espuma produzida foi espalhada sobre bandejas de aço inoxidável, compondo amostras com diferentes espessuras da camada de espuma $(0,5 ; 1,0$ e 1,5 cm), medidas com o auxilio de um paquímetro digital. As amostras nas bandejas foram levadas para secagem em estufa com circulação de ar, nas temperaturas de 50; 60 e $70^{\circ} \mathrm{C}$.

As cinéticas de secagem foram determinadas pesando-se as bandejas com as amostras, durante as secagens, até peso constante, em intervalos regulares. Com os dados experimentais foram calculados os valores da razão de umidade (Equação 1).

$$
R X=\frac{X-X_{\varepsilon}}{X_{0}-X_{\varepsilon}}
$$

Em que:

$\mathrm{RX}=$ razão de umidade, adimensional;

$X=$ umidade, base seca;

$X_{e}=$ umidade de equilíbrio, base seca;

$X_{0}=$ umidade inicial, base seca.

Os modelos semi-teóricos (Tabela 1) de Henderson e Pabis (Eq. 2), Henderson (Eq. 3) e Logarítmico (Eq. 4) foram utilizados para ajustar às curvas de secagem.

Tabela 1. Modelos matemáticos aplicados aos dados da cinética de secagem

\begin{tabular}{|c|c|c|c|}
\hline Modelo & Equação & & Referência \\
\hline Henderson \& Pabis & $R X=a \cdot \exp (-k t)$ & (2) & AGHBASHLO et al. (2009) \\
\hline Henderson & $R X=a \cdot \exp (-b t)+c \cdot \exp (-d t)$ & (3) & HENDERSON (1974) \\
\hline Logarítmico & $R X=a \cdot \exp (-k t)+c$ & (4) & DOYMAZ (2011) \\
\hline
\end{tabular}

Para avaliar o modelo que se ajusta melhor aos dados experimentais foram utilizados, como parâmetros o coeficiente de determinação $\left(R^{2}\right)$ e o desvio quadrático médio (Equação 5):

$$
D Q M=\sqrt{\frac{\sum\left(R X_{p r \varepsilon d}-R X_{\theta x p}\right)^{2}}{n}}
$$

Em que:

DQM - desvio quadrático médio;

$\mathrm{RX}_{\text {pred }}$ - razão de umidade predito pelo modelo;

$\mathrm{RX}_{\text {exp }}$ - razão de umidade experimental;

n - número de observações.

\section{Resultados e Discussão}

Na Figura 1 estão representadas as cinéticas de secagem em camada de espuma da polpa da manga Haden, elaborada com adição de 1,5\% de Super Liga Neutra ${ }^{\circledR}$ e 1,5\% de Emustab® durante 30 minutos de batimento, com espessuras da camada de espuma de 0,5; 1,0 e 1,5 cm e temperatura de secagem de 50; 60 e $70^{\circ} \mathrm{C}$, cujos dados são apresentados na forma de razão de umidade em função do tempo de secagem. No processo de secagem, notou-se que a espessura influenciou significativamente em todas as secagens ocorrendo de forma mais rápida nas espessuras menores; na temperatura de $70^{\circ} \mathrm{C}$ houve os menores tempos de secagem 480; 660 e 780 minutos para as espessuras de 0,$5 ; 1,0$ e $1,5 \mathrm{~cm}$, respectivamente; na temperatura de $60^{\circ} \mathrm{C}$ produziram-se os tempos de secagem intermediários de 630; 720 e 825 minutos, para as espessuras de 0,$5 ; 1,0$ e 1,5 $\mathrm{cm}$, respectivamente; a temperatura de $50^{\circ} \mathrm{C}$ resultou nos maiores tempos de secagem 690; 780 e 900 minutos, para as espessuras de 0,5; 1,0 e $1,5 \mathrm{~cm}$, respectivamente. Observa-se que a temperatura de secagem e a espessura da camada de espuma influenciaram no tempo de secagem.

Wilson et al. (2012) ao estudarem a secagem da polpa de manga da variedade Dussehri com adição de albumina (0, 3, 5, 7 e 9\%) em camada de espuma nas temperaturas de 65 ${ }^{\circ} \mathrm{C}$ (390 min), $75^{\circ} \mathrm{C}$ (330 min) e $85^{\circ} \mathrm{C}$ (300 min), também observaram que a temperatura do ar de secagem teve efeito significativo sobre o tempo de secagem, o que é evidente a partir do fato 
de que o tempo de secagem é reduzido com o aumento da temperatura do ar de secagem.
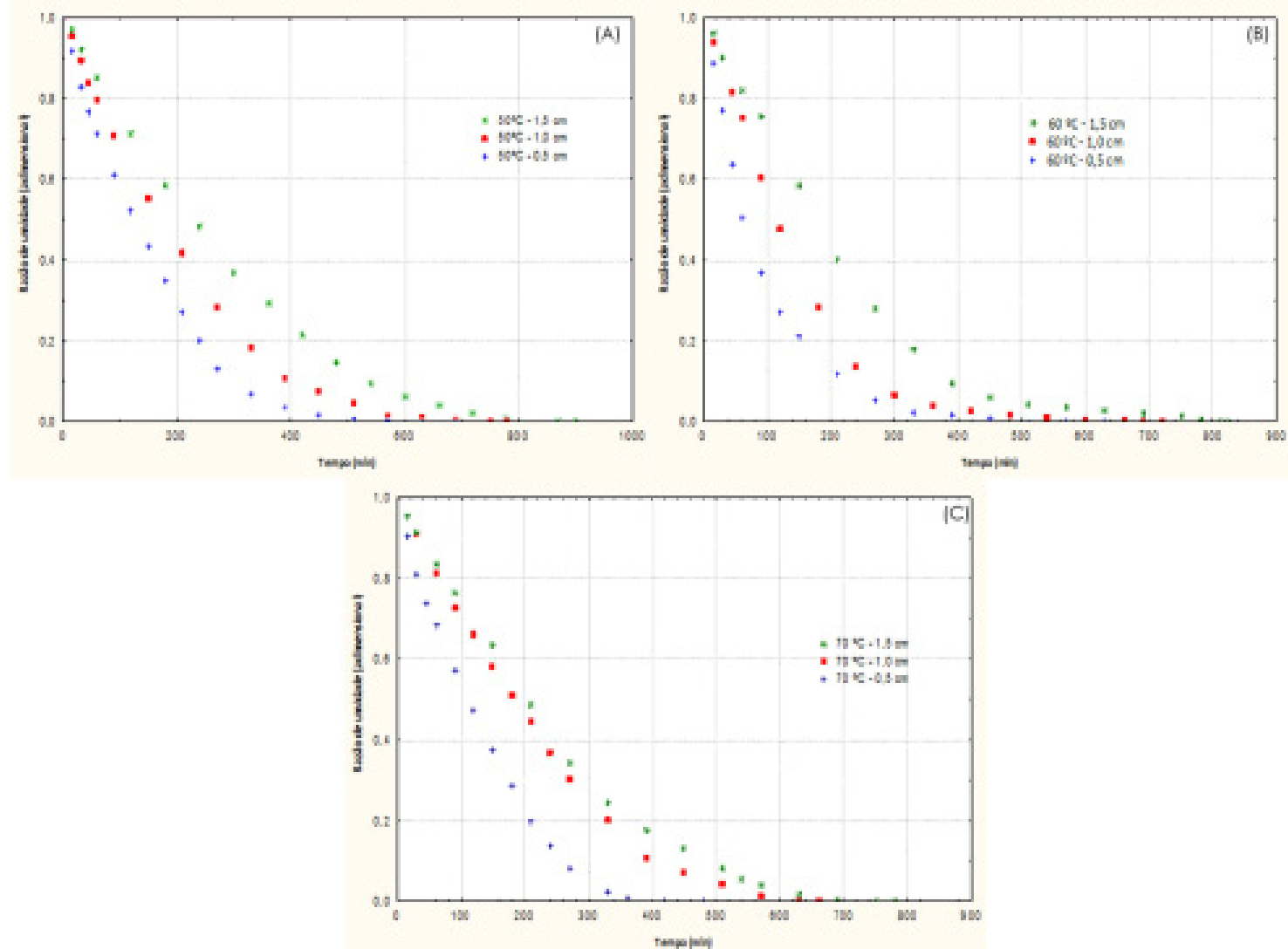

Figura 1. Curvas de cinética de secagem da formulação a 50 (A), 60 (B) e $70^{\circ} \mathrm{C}$ (C) para as espessuras de 0,$5 ; 1,0$ e $1,5 \mathrm{~cm}$ da camada de espuma

Rajkumar et al. (2007) avaliaram a cinética de secagem da polpa da manga Alphonso com aditivos (albumina e metilcelulose) em diferentes concentrações, nas temperaturas de secagem de $60,65,70$ e $75^{\circ} \mathrm{C}$ e espessuras da camada de espuma de 0,$1 ; 0,2$ e $0,3 \mathrm{~cm}$, verificando que a condição que reteve melhor as características bioquímicas foi para a formulação com $10 \%$ de albumina e 0,5\% de metilcelulose com uma espessura da camada de $0,1 \mathrm{~cm}$ e temperatura de $60^{\circ} \mathrm{C}$ secando a amostra em 40 minutos. Este tempo foi inferior ao encontrado para a polpa de manga cv. Haden do presente estudo. Kandasamy et al. (2014) ao avaliarem a secagem em camada de espuma da polpa de mamão com diferentes aditivos (metilcelulose, monoestearato de glicerol e albumina) em diferentes temperaturas $(60,65$ e $70^{\circ} \mathrm{C}$ ) e espessuras da camada de espuma $(0,2,0,4,0,6,0,8$ e 1,0 cm) também verificaram tendência de aumento do tempo de secagem com o aumento da espessura e redução do tempo com o aumento da temperatura, com os tempos variando entre 60 e $360 \mathrm{~min}$.

Observa-se que o tempo de secagem foi menor na temperatura de $70{ }^{\circ} \mathrm{C}$ quando comparando com a temperatura de $50^{\circ} \mathrm{C}$ para retirar a água contida na polpa de manga formulada, na ordem de aproximadamente $44 \%$, na espessura da camada de $0,5 \mathrm{~cm}$.

Verificam-se na Tabela 2 os valores dos parâmetros dos modelos matemáticos de Henderson \& Pabis, Henderson e Logarítmico, que ajustaram às curvas de secagem da formulação com polpa da manga Haden, desidratada em diferentes temperaturas de secagem (50, 60 e 70 ${ }^{\circ} \mathrm{C}$ ) e espessura da camada de espuma de 0,5 cm. Observa-se que todos os modelos testados podem representar o processo de secagem em razão de terem apresentado altos coeficientes de determinação $\left(R^{2}\right)$ sendo todos superiores a 0,97 e desvios quadráticos médios inferiores a 0,0036

Observa-se que dentre os modelos 
testados, o Logarítmico com três parâmetros, foi o que resultou nos melhores ajustes com maiores coeficientes de determinação e os menores desvios quadráticos médios, em todas as temperaturas.

Tabela 2. Parâmetros, coeficientes de determinação $\left(R^{2}\right)$ e desvios quadráticos médios (QDM) dos modelos ajustados às curvas de secagem da polpa de manga Haden, com espessura da camada de espuma de $0,5 \mathrm{~cm}$, para as diferentes temperaturas avaliadas

\begin{tabular}{|c|c|c|c|c|c|c|c|}
\hline \multirow{2}{*}{ Modelo } & \multirow{2}{*}{$\begin{array}{c}\text { Temperatura } \\
\left({ }^{\circ} \mathrm{C}\right) \\
\end{array}$} & \multicolumn{4}{|c|}{ Parâmetro } & \multirow{2}{*}{$\mathrm{R}^{2}$} & \multirow{2}{*}{ DQM } \\
\hline & & \multicolumn{2}{|l|}{$a$} & \multicolumn{2}{|c|}{$k$} & & \\
\hline \multirow{3}{*}{$\begin{array}{c}\text { Henderson \& } \\
\text { Pabis }\end{array}$} & 50 & \multicolumn{2}{|l|}{1,0912} & \multicolumn{2}{|c|}{0,0055} & 0,9744 & 0,0035 \\
\hline & 60 & \multicolumn{2}{|l|}{1,0622} & \multicolumn{2}{|c|}{0,0067} & 0,9806 & 0,0026 \\
\hline & 70 & \multicolumn{2}{|l|}{0,9927} & \multicolumn{2}{|c|}{$\begin{array}{l}0,0067 \\
0,0087\end{array}$} & 0,9908 & 0,0009 \\
\hline \multirow{4}{*}{ Logarítmico } & $\begin{array}{c}\text { Temperatura } \\
\left({ }^{\circ} \mathrm{C}\right)\end{array}$ & a & c & \multicolumn{2}{|r|}{$k$} & $R^{2}$ & DQM \\
\hline & 50 & 1,0984 & $-0,072$ & & 0,0041 & 0,9969 & 0,0004 \\
\hline & 60 & 1,1239 & $-0,081$ & & 0,0055 & 0,9878 & 0,0016 \\
\hline & 70 & 1,0331 & $-0,057$ & & 0,0077 & 0,9949 & 0,0005 \\
\hline \multirow{4}{*}{ Henderson } & $\begin{array}{c}\text { Temperatura } \\
\left({ }^{\circ} \mathrm{C}\right)\end{array}$ & a & $b$ & C & d & $\mathrm{R}^{2}$ & DQM \\
\hline & 50 & 0,522 & 0,004 & 0,52 & 0,004 & 0,9920 & 0,0012 \\
\hline & 60 & 0,531 & 0,006 & 0,53 & 0,006 & 0,9806 & 0,0026 \\
\hline & 70 & 0,495 & 0,008 & 0,49 & 0,008 & 0,9908 & 0,0009 \\
\hline
\end{tabular}

Adam \& Balasubramanian (2011), verificaram, em estudo de secagem em camada de espuma de suco de tomate com albumina $(0,5,10,15$ e 20\%), nas temperaturas de 60,65 e $70^{\circ} \mathrm{C}$, que o modelo Logarítmico foi o que melhor se ajustou aos dados experimentais apresentando os maiores $\mathrm{R}^{2}(0,985)$ e menores $\chi^{2}$. Constata-se que o parâmetro $\mathrm{k}\left(\mathrm{min}^{-1}\right)$, que é a constante de secagem, dos modelos de Henderson \& Pabis e Logarítmico, aumentou com o aumento da temperatura de secagem. Sousa et al. (2011) relataram que k representa o efeito das condições externas de secagem e que geralmente aumenta com a elevação da temperatura do ar de secagem. Comportamento semelhante foi observado por Silva et al. (2008) ao estudarem a cinética de secagem em camada de espuma da polpa de tamarindo cujo parâmetro $K\left(\mathrm{~min}^{-1}\right)$ para o modelo de Henderson \& Pabis também aumentou com o aumento da temperatura $\left(50,60\right.$ e $\left.70{ }^{\circ} \mathrm{C}\right)$; e por Melo et al. (2013) ao ajustarem este modelo as curvas de secagem em camada de espuma da polpa do fruto do mandacaru $\left(70,80\right.$ e $\left.90^{\circ} \mathrm{C}\right)$.

Apresentam-se na Figura 2, as cinéticas de secagem da formulação com polpa da manga Haden, com $0,5 \mathrm{~cm}$ de espessura da camada de espuma e desidratada em diferentes temperaturas de secagem $\left(50,60\right.$ e $\left.70^{\circ} \mathrm{C}\right)$, com ajuste pelo modelo de Logarítmico, considerado o melhor modelo entre os testados. Observa-se que as curvas ajustadas passam próximas aos pontos experimentais, indicando o bom ajuste do modelo.

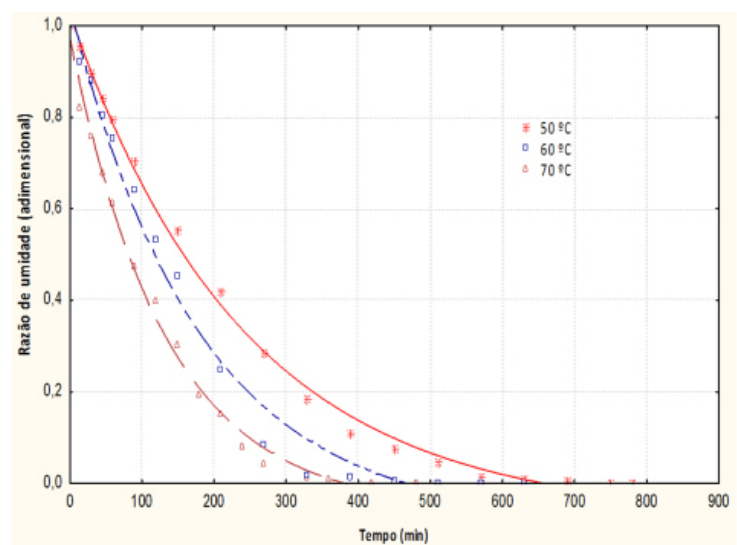

Figura 2. Cinética de secagem da polpa da manga Haden, com espessura da camada de espuma de $0,5 \mathrm{~cm}$ nas diferentes temperaturas, com ajuste pelo modelo de Logarítmico.

Apresentam-se na Tabela 3, os valores dos parâmetros dos modelos matemáticos de Henderson \& Pabis, Logarítmico e Henderson, ajustados as curvas de secagem da formulação com polpa da manga Haden, desidratada em diferentes temperaturas de secagem $(50,60 \mathrm{e}$ $70^{\circ} \mathrm{C}$ ) com 1,0 cm de espessura da camada de espuma, os coeficientes de determinação $\left(R^{2}\right)$ e o desvios quadráticos médios. Observa-se que todos os modelos podem representar o processo de secagem em razão de terem apresentados coeficientes de determinação $\left(R^{2}\right)$ superiores a 0,97 e desvios quadráticos médios inferiores a 
0,003. Dentre os modelos testados, o Logarítmico foi o que resultou nos melhores ajustes com os maiores coeficientes de determinação $\mathrm{R}^{2}>$
0,98, e os menores desvios quadráticos médios, inferiores ou iguais a 0,0004.

Tabela 3. Parâmetros, coeficientes de determinação $\left(R^{2}\right)$ e desvios quadráticos médios (QDM) dos modelos ajustados às curvas de secagem da polpa de manga Haden, com espessura da camada de espuma de 1,0 cm, para as diferentes temperaturas avaliadas

\begin{tabular}{|c|c|c|c|c|c|c|c|}
\hline \multirow{2}{*}{ Modelo } & \multirow{2}{*}{$\begin{array}{c}\text { Temperatura } \\
\left({ }^{\circ} \mathrm{C}\right)\end{array}$} & \multicolumn{4}{|c|}{ Parâmetro } & \multirow{2}{*}{$\mathrm{R}^{2}$} & \multirow{2}{*}{ DQM } \\
\hline & & & & & $k$ & & \\
\hline \multirow{3}{*}{$\begin{array}{c}\text { Henderson \& } \\
\text { Pabis }\end{array}$} & 50 & \multicolumn{2}{|c|}{1,0467} & \multirow{2}{*}{\multicolumn{2}{|c|}{0,0001}} & 0,9809 & 0,0024 \\
\hline & 60 & \multicolumn{2}{|c|}{1,0440} & & & 0,9961 & 0,0005 \\
\hline & 70 & \multicolumn{2}{|c|}{1,0238} & \multicolumn{2}{|r|}{0,0084} & 0,9967 & 0,0004 \\
\hline \multirow{4}{*}{ Logarítmico } & $\begin{array}{c}\text { Temperatura } \\
\left({ }^{\circ} \mathrm{C}\right)\end{array}$ & \multicolumn{2}{|l|}{ a } & \multicolumn{2}{|r|}{ k } & $\mathrm{R}^{2}$ & $D Q M$ \\
\hline & 50 & \multicolumn{2}{|l|}{1,098} & 072 & 0,0041 & 0,9968 & 0,0004 \\
\hline & 60 & \multicolumn{2}{|l|}{1,065} & 032 & 0,0052 & 0,9977 & 0,0003 \\
\hline & 70 & 1,033 & \multicolumn{2}{|c|}{$-0,014$} & 0,0080 & 0,9973 & 0,0003 \\
\hline \multirow{4}{*}{ Henderson } & $\begin{array}{c}\text { Temperatura } \\
\left({ }^{\circ} \mathrm{C}\right)\end{array}$ & a & $b$ & C & d & $\mathrm{R}^{2}$ & DQM \\
\hline & 50 & 0,53 & 0,003 & 0,510 & 0,003 & 0,9808 & 0,0024 \\
\hline & 60 & 0,52 & 0,005 & 0,521 & 0,005 & 0,9961 & 0,0004 \\
\hline & 70 & 0,91 & 0,008 & 0,113 & 0,008 & 0,9967 & 0,0003 \\
\hline
\end{tabular}

Comportamento semelhante foi encontrado por Ambekar et al. (2013) ao empregarem o modelo de Henderson \& Pabis as curvas de cinética de secagem em camada de espuma da polpa de maracujá com adição de metilcelulose (3\%) nas temperaturas de 50 , 60, 70 e $80^{\circ} \mathrm{C}$ encontraram $\mathrm{R}^{2}$ entre 0,98-0,99 e $\chi^{2}$ entre 0,00013-0,00053. BABALIS et al. (2006) ao estudarem a secagem do figo nas temperaturas de 55 a $85^{\circ} \mathrm{C}$ encontraram, para o modelo de Henderson \& Pabis, $R^{2}>0,990$ para todas as temperaturas de secagem.

Nota-se, no modelo Logarítmico, que o parâmetro k aumentou quando a temperatura aumentou de 50 para $70^{\circ} \mathrm{C}$; resultado semelhante ao encontrado no trabalho de ALEXANDRE et al. (2013), em estudo da cinética de secagem do resíduo de abacaxi enriquecido, tendo sido constatado que o parâmetro K, que representa a constante da taxa de secagem, aumentou com o aumento da temperatura quando se analisam as temperaturas entre 40 e $60^{\circ} \mathrm{C}$.

$\mathrm{Na}$ Figura 3 se apresentam as cinéticas de secagem da formulação com polpa da manga Haden, com $1,0 \mathrm{~cm}$ de espessura da camada de espuma desidratada em diferentes temperaturas de secagem $\left(50,60\right.$ e $\left.70^{\circ} \mathrm{C}\right)$, com ajuste pelo modelo Logarítmico, considerado o melhor modelo entre os testados; verifica-se que as curvas ajustadas ficaram próximas aos dados experimentais.

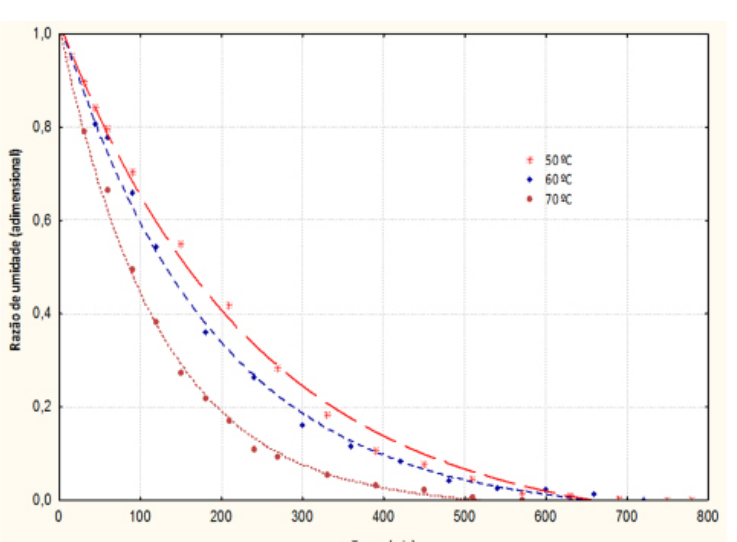

Figura 3. Cinética de secagem da polpa da manga Haden, com espessura da camada de espuma de $1,0 \mathrm{~cm}$, nas diferentes temperaturas, com ajuste pelo modelo de Logarítmico.

Na Tabela 4 são apresentados os valores dos parâmetros dos modelos matemáticos de Henderson \& Pabis, Logarítmico e Henderson ajustados as curvas de secagem da formulação com polpa da manga Haden, desidratada em diferentes temperaturas de secagem $(50,60$ e 70 $\left.{ }^{\circ} \mathrm{C}\right)$, com $1,5 \mathrm{~cm}$ de espessura da camada de espuma, os coeficientes de determinação $\left(R^{2}\right)$ e o desvios quadráticos médios. Observa-se que todos os modelos testados podem representar o processo de secagem em razão de terem apresentados coeficientes de determinação $\left(R^{2}\right)$ superiores a 0,98 e desvios quadráticos médios inferiores a 0,0026.

Verifica-se que dentre os modelos testados o que resultou no melhor ajuste, com 
- maior $\mathrm{R}^{2}$, na temperatura de $50{ }^{\circ} \mathrm{C}$ foi $\mathrm{O}$ Logarítmico, na temperatura de $60^{\circ} \mathrm{C}$ foi o de Henderson e na temperatura de $70{ }^{\circ} \mathrm{C}$ foi o de Henderson \& Pabis. Comportamento similar foi encontrado por Silva et al. (2008) em estudo de secagem de polpa de tamarindo em camada de espuma, nas temperaturas de 60 e $70{ }^{\circ} \mathrm{C}$ encontrando valor de $R^{2}>0,99$ com o modelo de Henderson \& Pabis.

Observa-se que o parâmetro $k$ dos modelos de Henderson \& Pabis aumentou com - aumento da temperatura, comportamento também observado por Kaya et al. (2007) ao utilizarem o modelo de Henderson \& Pabis para descrever a secagem de maça nas temperaturas de 35,45 e $55^{\circ} \mathrm{C}$.

$\mathrm{Na}$ Figura 4 verificam-se as cinéticas de secagem da polpa da manga Haden, desidratada com $1,5 \mathrm{~cm}$ de espessura da camada de espuma e em diferentes temperaturas de secagem $\left(50,60\right.$ e $\left.70^{\circ} \mathrm{C}\right)$ com ajustes pelos modelos Logarítmico $\left(50^{\circ} \mathrm{C}\right)$, Henderson $\left(60^{\circ} \mathrm{C}\right)$ e Henderson \& Pabis $\left(70^{\circ} \mathrm{C}\right)$, que foram os que apresentaram os melhores ajustes aos dados experimentais. Observa-se que as curvas ajustadas ficaram próximas aos dados experimentais.

Tabela 4. Parâmetros, coeficientes de determinação $\left(R^{2}\right)$ e desvios quadráticos médios (QDM) dos modelos ajustados às curvas de secagem da polpa de manga Haden, com espessura da camada de espuma de $1,5 \mathrm{~cm}$, para as diferentes temperaturas avaliadas

\begin{tabular}{|c|c|c|c|c|c|c|c|}
\hline \multirow{2}{*}{ Modelo } & \multirow{2}{*}{$\begin{array}{c}\text { Temperatura } \\
\left({ }^{\circ} \mathrm{C}\right)\end{array}$} & \multicolumn{4}{|c|}{ Parâmetro } & \multirow{2}{*}{$\mathrm{R}^{2}$} & \multirow{2}{*}{ DQM } \\
\hline & & $a$ & & $\mathrm{k}$ & & & \\
\hline \multirow{3}{*}{$\begin{array}{c}\text { Henderson \& } \\
\text { Pabis }\end{array}$} & 50 & 1,0467 & \multicolumn{3}{|c|}{0,0037} & 0,9903 & 0,0025 \\
\hline & 60 & 1,0600 & \multicolumn{3}{|c|}{0,0049} & 0,9942 & 0,0016 \\
\hline & 70 & 1,0018 & \multicolumn{3}{|c|}{0,0072} & 0,9991 & 0,0002 \\
\hline \multirow{4}{*}{ Logarítmico } & $\begin{array}{c}\text { Temperatura } \\
\left({ }^{\circ} \mathrm{C}\right)\end{array}$ & a & \multicolumn{2}{|c|}{ c } & k & $R^{2}$ & DQM \\
\hline & 50 & 1,1612 & \multicolumn{2}{|c|}{$-0,140$} & 0,0027 & 0,9928 & 0,0009 \\
\hline & 60 & 1,0976 & \multicolumn{2}{|c|}{$-0,052$} & 0,0043 & 0,9923 & 0,0011 \\
\hline & 70 & 1,0069 & \multicolumn{2}{|c|}{0,001} & 0,0072 & 0,9983 & 0,0002 \\
\hline \multirow{4}{*}{ Henderson } & Temperatura & a & $b$ & C & d & $R^{2}$ & DQM \\
\hline & 50 & 0,522 & 0,003 & 0,5241 & 0,003 & 0,9808 & 0,0024 \\
\hline & 60 & $-23,9$ & 0,008 & 24,963 & 0,008 & 0,9985 & 0,0002 \\
\hline & 70 & 0,529 & 0,008 & 0,4754 & 0,006 & 0,9983 & 0,0002 \\
\hline
\end{tabular}

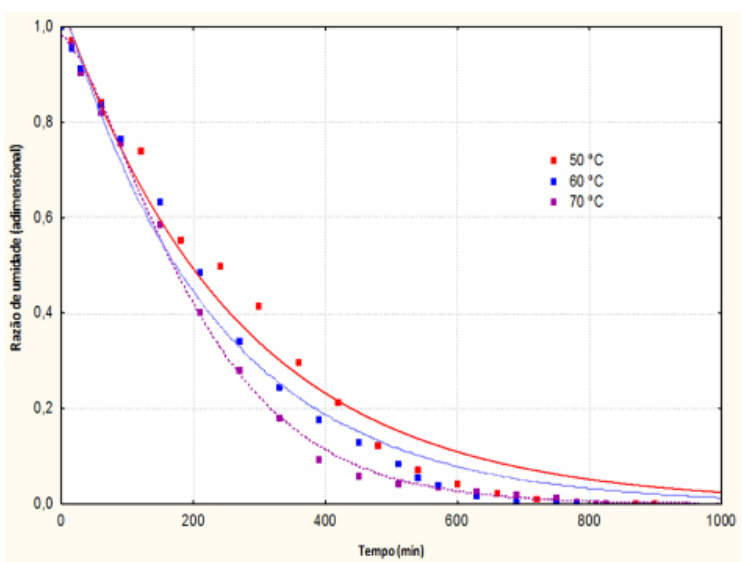

Figura 4. Cinética de secagem da polpa da manga Haden, com espessura da camada de espuma de $1,5 \mathrm{~cm}$, nas diferentes temperaturas, com ajustes pelos modelos Logarítmico $\left(50^{\circ} \mathrm{C}\right)$, Henderson $\left(60^{\circ} \mathrm{C}\right)$ e Henderson \& Pabis $\left(70^{\circ} \mathrm{C}\right)$.

Bons ajustes também foram encontrados por Ferreira et al. (2012) em estudo com secagem em camada delgada de bagaço de uva fermentado, nas temperaturas de 50,60 e $70^{\circ} \mathrm{C}$, que obtiveram coeficientes de determinação superiores a 0,99 e desvios quadráticos médios inferiores a 0,01 para o modelo de Henderson e Pabis e Logarítmico.

\section{Conclusões}

Dos experimentos de secagens realizados foi observado que quanto menor a espessura da camada de espuma e maior a temperatura do ar de secagem menor é o tempo desecagem; e os modelos avaliados de Henderson \& Pabis, Logarítmico e Henderson podem ser utilizados na estimativa das curvas de secagem em camada de espuma da polpa da manga cv. Haden, com o modelo Logarítmico na maioria dos casos descrevendo melhor as curvas.

\section{Referências}

Aghbashlo, M., Kianmehr, M.H., Arabhosseini, A., 
Nazghelichi, T. 2009. Mathematical modelling of thin-layer drying of carrot. International Agrophysics 23: 313-317.

Alexandre, V.A., Silva, F.L.H., Gomes, J.P., Silva, O.S., Carvalho, J.P.D., Lima, E.E. 2013. Cinética de secagem do resíduo de abacaxi enriquecido. Revista Brasileira de Engenharia Agrícola 17: 640646.

Ambekar, S.A., Gokhale, S.V., Lele, S.S. 2013. Process optimization for foam mat-tray drying of Passiflora edulis Flavicarpa pulp and characterization of the dried powder. International Journal of Food Engineering 9: 433443.

Babalis, S.J., Papanicolaou, E., Kyriakis, N., Belessiotis, V.G. 2006. Evaluation of thin-layer drying models for describing drying kinetics of figs (Ficus carica). Journal of Food Engineering, 75: 205-214.

Costa, J.M.C., Medeiros, M.F.D., Mata, A.L.M.L. 2003. Isotermas de adsorção de pós de beterraba (Beta vulgaris L.), abóbora (Cucurbita moschata) e cenoura (Daucus carota) obtidos pelo processo de secagem em leito de jorro: estudo comparativo. Revista Ciência Agronômica 34: 5-9.

Doymaz, I. 2011. Sun drying of seedless and seeded grapes. Journal of Food Science and Technology 49: 214-220.

El-Aouar, A.A., Murr, F.E.X. 2003. Estudo e modelagem da cinética de desidratação osmótica do mamão formosa (Carica papaya L.). Ciência e Tecnologia de Alimentos 23: 69-75.

Femenia, A., Sastre-Serrano, G., Simal, S., Garau, M. C., Eim, V. S., Rosselló, C. 2009. Effects of airdrying temperature on the cell walls of kiwifruit processed at different stages of ripening. LWT Food Science and Technology 42:106-112.

Ferreira, L.F.D., Pirozi, M.R., Ramos, A.M., Pereira, J.A.M., 2012. Modelagem matemática da secagem em camada delgada de bagaço de uva fermentado, Agropecuária Brasileira, 47: 855-862.

Henderson, S.M. 1974. Progress in developing the thin layer drying equation. Transactions of the ASAE 17: 1167-1168.

IBGE. Instituto Brasileiro de Geografia e Estatística. Produção Agrícola Municipal. 2011. IBGE, Rio de Janeiro, Brasil. $465 \mathrm{p}$.

IBGE. Instituto Brasileiro de Geografia e Estatística. Anuário Estatístico do Brasil. 2013. IBGE, Rio de Janeiro, Brasil. $467 \mathrm{p}$.

Kadam, D.M., Balasubramanian, S. 2011. Foam mat drying of tomato juice. Journal of Food Processing and Preservation 35: 488-495.

Kandasamy, P., Varadharaju, N., Kalemullah, S., Maladhi, D. 2014. Optimization of process parameters for foam-mat drying of papaya pulp. Journal of Food Science and Technology $51: 2526-2534$.

Kaya, A., Aydin, O., Demirtas, C. 2007. Drying kinetics of red delicious apple. Biosystems Engineering 96: 517-524.

Kuskoski, E.M., Asuero, A.G., Morales, M.T., Fett, R. 2006. Frutos tropicais silvestres e polpas de frutas congeladas: atividade antioxidante, polifenóis e antocianinas. Ciência Rural 36: 1283-1287.

Melo, K.S., Figueirêdo, R.M.F., Queiroz, A.J.M., Fernandes, T.K.S., Bezerra, M.C.T. 2013. Secagem em camada de espuma da polpa do fruto do mandacaru: Experimentação e ajustes de modelos matemáticos. Revista Caatinga 26: 1017.

Rajkumar, P., Kailappan, R., Viswanathan, R., Raghavan, G.S.V. 2007. Drying characteristic of foamed Alphonso mango pulp in a continuous type foam mat dryer. Journal of Food Engineering 79: 1452-1459.

Silva, A.S., Gurjão, K.C.O., Almeida, F.A.C., Bruno, R.L.A., Pereira, W.E. 2008. Dehydration of tamarind pulp through the foam-mat drying method. Ciência e Agrotecnologia 32: 1899-1905.

Sousa, K.A., Resende, O., Chaves, T.H., Costa, L.M. 2011. Cinética de secagem do nabo forrageiro (Raphanus sativus L.). Revista Ciência Agronômica 42: 883-892.

Wilson, R.A., Kadam, D.M., Chadha, S., Sharma, $M$. Foam mat drying characteristics of mango pulp. 2012. International Journal of Food Science and Nutrition Engineering 2: 63-69. 